\title{
Capacity Enhancement of MIMO-OFDM System in Rayleigh Fading Channel
}

\author{
Atul Gautam ${ }^{1}$, Manisha Sharma ${ }^{2}$ \\ ${ }^{1}$ Department of Electronics \& Communication, M.Tech Scholar, Lovely professional university, Punjab, India \\ ${ }^{2}$ Department of Electronics \& Communication, Faculty of Electronics \& Communication Engineering, Punjab, \\ India
}

\begin{abstract}
MIMO-OFDM system in Rayleigh Fading Channel is very popular technique for mobile communication now a day's for research. Here we want increase the capacity of MIMO-OFDM of system by using adaptive modulation, Algebraic Space-Time Codes (ASTC) encoder for MIMO Systems are based on quaternion algebras .we found that ergodic capacity has some limitation which reduce the system's performance to overcome this we use ASTC code. ASTC code are full rank, full rate and non vanishing constant minimum determinant for increasing spectral efficiency and reducing Peak to Average Power Ratio (PAPR). Keywords-Adaptive modulation ASTC code, Capacity, BER, Ergodic capacity, PAPR, Spectral Efficiency and SNR
\end{abstract}

\section{INTRODUCTION}

NOW A day's integration of Orthogonal Frequency division Multiplexing (OFDM) technique with Multiple Input Multiple Output (MIMO) systems has been an area of interesting and challenging research in the field of broadband wireless communication. Multiple input multiple output (MIMO) system using multiple transmit and receive antennas are widely recognized as the vital breakthrough that will allow future wireless systems to achieve higher data rates with limited bandwidth and power resources, provided the propagation medium is rich scattering or Rayleigh fading[1].On the other hand, traditionally, multiple antennas have been used to increase diversity to combat channel fading. Hence, A MIMO system can provide two types of gains: spatial multiplexing or capacity gain and diversity gain. If we need to use the advantage of MIMO diversity to overcome the fading then we need to send the same signals through the different MIMO antennae. If we want to use MIMO concept for increasing capacity then we need to send different set of data at the same time through the different MIMO antennae without the automatic-repeat request of the transmission [2]. OFDM has many advantages, which make it an attractive scheme for high-speed transmission links. However, one major difficulty is OFDM's large Peak to Average Power Ratio (PAPR). Those are created by the coherent summation of the OFDM subcarriers. When $\mathrm{N}$ signals are added with the same phase, they produce a peak power that is $\mathrm{N}$ times the average power. These large peaks cause saturation in the power amplifiers, leading to inter modulation products among the subcarrier and disturbing out of band energy. Hence, it becomes worth while reducing PAPR. Towards this end there are several proposals such as clipping, coding and peak windowing. Respectively, reduction of PAPR comes at a price of performance degradation, mainly in terms of rate and BER. This paper proposes to use the ASTC codes as powerful coding techniques for IEEE 802.11x OFDM standard combined with PAPR scheme [7]. ASTC codes can for out a good solution first to overcome the disadvantage of OFDM modulations and second to keep a robustness regarding the BER performances. ASTC encoder is shaped from two well known algebraic space time codes. The first one is called the Golden code (GC), which was proposed in 2004. It is a $2 \times 2$ STBC obtained using a division algebra, which is full rate, full diversity, and has a nonzero lower bound on its coding gain, which does not depend on the constellation size. The second code is the TAST code (TC) a2 $\times 2$ space time algebraic code obtained using the integer algebra, with rate $R=n_{t}=2$ Symbol/uc, and diversity $D=n_{t} \times n_{r}=4$, where uc denotes the used code word [6].Adaptive modulation is a promising technique to increase the spectral efficiency of a wireless communication system. In this paper we investigate the effectiveness of adaptive modulation in maximizing the spectral efficiency of a MIMO multiuser downlink channel [11].Under an average transmit power and instantaneous bit error rate (BER) constraint, the transmit parameters including the sub channel transmit power and/or spectral efficiency are optimally adapted in the spatial and/or temporal domain to maximize the average spectral efficiency (ASE). Two categories, the continuous rate and discrete rate, of adaptive systems were considered. In the continuous rate category, we first consider the ASE optimization problem with both power and spectral efficiency to be jointly adapted, which is referred to as a variable rate variable power (VRVP) system. The optimal power and rate adaptation policy, as well as the ASE expression, are derived. Following that, two special cases are studied, the variable rate (VR) system and the 
variable power (VP) system. The VR system fixes the power as constant while the VP system fixes the rate. The closed form asymptotic expressions for the ASEs of these three adaptive systems are derived. The asymptotic ASEs for VRVP and VR systems are the same and both achieve a full multiplexing gain. Compared with the VRVP and VR systems, the asymptotic ASE for a VP system with different numbers of transmit and receive antennas shows a constant signal-to-noise ratio (SNR) penalty, while a VP system equipped with the same number of transmit and receive antennas is unable to achieve the full multiplexing gain. In the discrete rate category, the power and rate adaptation policy and the ASE are also derived for VRVP, VR, and VP systems. It is shown that the ASE results for the continuous rate systems act as tight upper bounds for their discrete rate counterparts. In particular, for the discrete rate VR system, we obtained a closed form expression for the ASE and show that there is a 2-3 dB SNR penalty compared to the continuous rate counterpart. However, the advantages include a much simpler adaptation rule, a better BER performance, and a preserved full multiplexing gain [6]. We will refer to this class of schemes as adaptive QAM (A-QAM) with the following nomenclature. We say an A-QAM scheme is XY-Z-L for X and Y representing the type of variation for rate (equivalently, constellation size) and power, respectively. Three options are possible for this variation: ' $C$ ' (Continuous), 'D' (Discrete) and 'K' (Constant). The Z corresponds to the type of BER constraint, which can be 'I' (Instantaneous) or A' (Average). Finally, for discrete-power schemes, L is the allowed number of power levels per constellation [11].The paper is organized as follows. Section II, system model is described. Section III, ASTC encoder is described. Section IV, frequency-selective correlated rayleigh fading channel .Section V, Adaptive modulation is described. In section VI, we present simulation result for different scenarios. Finally, a conclusion is given in section VII.

\section{System Model}

A model of MIMO-OFDM system with $\mathrm{N}_{\mathrm{Tx}}$ transmit antennas and $\mathrm{N}_{\mathrm{Rx}}$ receive antennas is depicted in the Figure 1. Let, $x_{i}, y_{i}$ and $r_{i}$ be the transmitted signal, received signal and the Additive White Gaussian Noise (AWGN) for the $\mathrm{I}^{\text {th }}$. The sub-carrier respectively and the system uses frequency selective channel. Then the received signal can be given as,

$\mathrm{Y}_{\mathrm{i}}=\mathrm{HiSi}+\mathrm{r}_{\mathrm{i}} ; \quad 0 \leq \mathrm{i} \leq \mathrm{N} \mathrm{S}$

In Eq. (1), Ns represent the number of sub -carriers $H_{i}$ is the channel response matrix of $I^{\text {th }}$ the sub-carrier that is of size $\mathrm{N}_{T x} * \mathrm{~N}_{\mathrm{Rx}}$. The $\mathrm{H}_{\mathrm{i}}$ is a Gaussian random matrix whose realization is known at the receiver and it is given as

$$
H_{i}=\sum_{l=0}^{L-1} h_{l} \exp \left(-\mathrm{j} * 2 \pi * \mathrm{i} * 1 / \mathrm{N}_{S}\right)
$$

In Eq. (2) hl is assumed to be an uncorrelated channel matrix where each element of the matrix follows the independently and identically distributed (IID) complex Gaussian distribution and L represents the tap of the chosen channel (i.e. L-tap frequency selective channel). It is assumed that a perfect channel state information (CSI) is available at the receiver but not at the transmitter. The total available power is also assumed to be allocated uniformly across all space-frequency sub-channels.

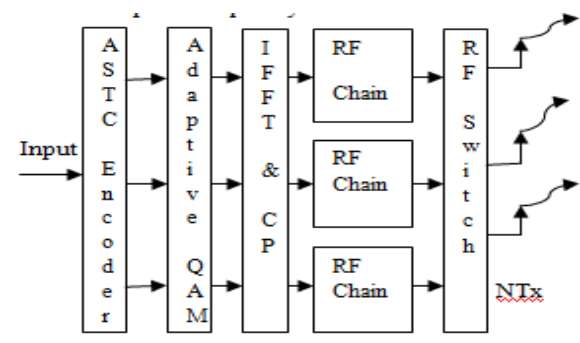

(a) Transmitter

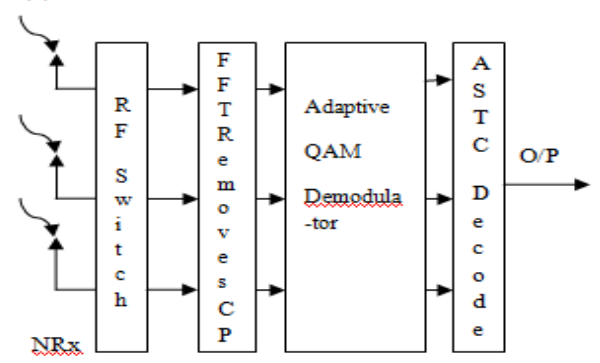

Fig 1: Block Diagram of MIMO-OFDM system (a) Transmitter and (b) Receiver 
In MIMO-OFDM system Ergodic capacity is define as this is the time average capacity of a channel. It is found by taking the mean of the capacity values obtained from a number of independent channel realizations. Ergodic capacity is define by equation

$$
c=E\left(\frac{1}{N_{S}} \sum_{i=0}^{N_{S}-1} \log \left(\left|I_{N_{R x}}+\gamma \cdot \mathrm{Q}\right|\right)\right)
$$

Where $\gamma=\frac{\rho}{n_{T x}}$

$$
Q=H_{i} H_{i}^{H}
$$

In above equation $\mathrm{E}$ (.) denotes the Ergodic capacity and $\mathrm{I}_{\mathrm{NRx}}$ is identity matrix of $\mathrm{N}_{\mathrm{Rx}} * \mathrm{~N}_{\mathrm{Rx}}$. $\mathrm{P}$ is $\mathrm{SNR}$ per sub carrier $\mathrm{N}_{\mathrm{Tx}}$ is number of transmit antenna .figure 1 shows the block diagram of MIMO-OFDM system. We use ASTC Encoder and Adaptive QAM (Quaderature Amplitude Modulation) for transmission. CP (Control Programming) is an operating system originally created for 8 bit processor. FFT is an efficient algorithm to compute the discrete Forier transform and its inverse.RF switch generally called Radio Frequency switch. PIN Diode is generally used to make it operate at very high frequency. In this switch input signal is fed at one end then this signal is split in no of output signal by demux. [1, 3]

\section{AStC Codes In A Frequency-Selective Channel ConTeXT}

We consider a coherent system over a frequency-selective correlated Rayleigh fading MIMO channel. The overall schematic diagram of ASTC-MIMO-OFDM transceiver is depicted in Fig.1.The transmitted binary source sequence bi of length $\mathrm{L}$ is modulated using the adaptive QAM-4 modulator. Each information sequence at time $\mathrm{n}_{\mathrm{i}}$.

$$
\vartheta_{n_{i}, n_{i+1}}=\left[S_{\left(2 \mathrm{n}_{i}+1\right)}-3, S_{\left(2 \mathrm{n}_{i}+1\right)}-2, S_{\left(2 \mathrm{n}_{i}+1\right)}-1, S_{\left(2 \mathrm{n}_{i}+1\right)}\right]^{T}
$$

Is encoded

by the ASTC encoder into two stream constellations represented by the code word $\mathrm{X}_{\mathrm{Nc}^{*} \mathrm{Nt}}$ where $\mathrm{N}_{\mathrm{t}}$ refers to number of transmitted antennas and $\mathrm{Nc}$ is the number of used subcarriers. By their construction the channel was under the Quasi-Static

Assumption, and does take into account neither the time variation nor the selectivity channel case. To spread their power regarding the bit rate and the BER performance into the selective channel case with time variation, we introduce the best perfect algebraic code known as Golden codes with other tow well famous algebraic space time codes, TAST and DAST [6].

\section{A. Golden Encoder}

The code was proposed in 2004 by a STBC obtained using a division algebra, which is full rate, full diversity, and has a nonzero lower bound on its coding gain, which does not depend on the constellation size. The code word is written as:

$$
X_{n_{i}}=\frac{1}{\sqrt{5}}\left(\begin{array}{ll}
\alpha\left(\vartheta_{n_{i}}(1)+\theta \vartheta_{n_{i}}(2)\right) & \alpha\left(\vartheta_{n_{i}}(3)+\theta \vartheta_{n_{i}}(4)\right) \\
\bar{\alpha}\left(\vartheta_{n_{i}}(3)+\bar{\theta} \vartheta_{n_{i}}(4)\right) & \bar{\alpha}\left(\vartheta_{n_{i}}(1)+\bar{\theta} \vartheta_{n_{i}}(2)\right)
\end{array}\right)
$$

Where

$$
\theta=\frac{1+\sqrt{5}}{2} \text { And } \bar{\theta}=\frac{1-\sqrt{5}}{2}, \frac{\alpha}{\alpha}=1+i-i \theta
$$

\section{B. TAST Encoder}

As shown in [7] [9], the TAST code is a space time algebraic code obtained using the integer algebra, with rate $\mathrm{R}=\mathrm{Nt}=2$ Symbole/uc (used code word), and diversity $\mathrm{D}=\mathrm{Nt} \times \mathrm{Nr}=4$. Each space time layer is associated with his proper algebraic space ' in order to alleviate the problem of ISI (Inter-Symbol-Interferences). The code word is expressed as:

$$
X_{n_{i}}=\frac{1}{\sqrt{2}}\left(\begin{array}{cc}
(\vartheta(1)+\theta \vartheta(2)) & \varphi(\vartheta(3)+\theta \vartheta(4)) \\
\varphi(\vartheta(3)+\theta \vartheta(4)) & (\vartheta(1)+\theta \vartheta(2))
\end{array}\right)
$$

Where, 
$\theta=\exp (\mathrm{i} \lambda)$

$\lambda \in \mathfrak{R}$

$\varphi=\theta^{2}$

\section{DAST Encoder}

The DAST code is a diagonal space time algebraic code obtained using the turned constellations of integer algebra, with rate 1 Symbole/uc, and full diversity. The code word is described as'

$$
\begin{aligned}
& X_{\text {Dast }}=H_{n t .} \operatorname{diag}\left(\mathrm{M} \vartheta_{n t}\right) \\
& \mathrm{M}=\frac{1}{\sqrt{2}}\left(\begin{array}{cc}
1 & \theta \\
1 & -\theta
\end{array}\right) \\
& \theta=\exp (\mathrm{i} \pi / 4)
\end{aligned}
$$

$M$ is the rotation matrix of $n_{t}=2$ degree. $[7,8]$

\section{Frequency-Selective Correlated Rayleigh Fading Channel}

Wide-Band systems are commonly a Frequency-Selective Correlated Rayleigh Fading Channels. However the ASTC requires a nonselective flat fading channels belonging to narrow-band systems. To alleviate this problem let focus on lattice representation of a Frequency-Selective Correlated Rayleigh Fading Channels. We adopt here the Clarke channel model. The received signal is the sum of q waves; we take into account the Doppler shifts effect. To obtain a correlated Rayleigh fading channel, the autocorrelation function of $\left\{h_{k}{ }^{j}\right\}$ process is given by:

$$
\begin{aligned}
& r_{h}=E\left[\mathrm{~h}_{k}^{i} \mathrm{~h}_{k-q}^{j}\right] \\
& r_{h}=\sigma^{2} \exp \left(\mathrm{j} 2 \pi \mathrm{f}_{c} \mathrm{q}\right) \mathrm{j}_{0}\left(2 \pi \mathrm{f}_{m} \mathrm{qT}_{s}\right)
\end{aligned}
$$

Where $J_{o}$ is the Bessel function with zero order, $f_{m}$ is the maximum Doppler shift and $j$ is the antenna's number. If we guess that we have $\mathrm{Nt}(\mathrm{Nc}+\mathrm{Ng})$ subcarrier used and the channel length is $\mathrm{L} \leq \mathrm{Nt}(\mathrm{Nc}+\mathrm{Ng})$ we can represent the channel in function of the correlated Rayleigh taps $h_{k}$, where $\mathrm{Ng}$ refers to the number of guard subcarrier and $\mathrm{Nt}$ to the number of transmitted antennas as follows

$$
\boldsymbol{H}=\left(\begin{array}{cccccccc}
h_{0} & 0 & \ldots & \ldots & \ldots & \ldots & \ldots & 0 \\
h_{1} & \ddots & & & \vdots & & & \vdots \\
h_{2} & & \ddots & \ldots & 0 & \ldots & & \vdots \\
\vdots & & & \ddots & \vdots & & & \vdots \\
h_{L-1} & \ldots & \ldots & \ldots & h_{0} & & & \vdots \\
0 & \ddots & & & & \ddots & & \vdots \\
\vdots & & \ddots & & & & \ddots & 0 \\
0 & \ldots & \ldots & h_{L-1} & \ldots & \ldots & \ldots & h_{0}
\end{array}\right)
$$

In order to use the ASTC codes properly, we need to convert the channel $\mathrm{H}$ into $\mathrm{N}_{\mathrm{t}} \times(\mathrm{Nc}+\mathrm{Ng})$ non selective sub-channels, The core idea is that the wide-band frequency selective MIMO channel by means of the MIMOOFDM processing is transferred to a number of parallel flat fading MIMO channels. In fact each code word xp ni will be modulated within the NcNt sub-channels, without loss of generality, now we are assuming that all subcarriers are used:

$$
z=\sqrt{N_{c}}\left(F^{-1} \otimes I_{N t}\right) \mathrm{x}^{p} N_{c} N_{t, 1}
$$

This transforms the frequency domain vector $\mathrm{x}_{\mathrm{NcNt}}, 1$ into the time domain. Where $\mathrm{x}$ represents the Kronecker product and $\mathrm{F}-1$ represent the IFFT Matrix defined as:

$$
\boldsymbol{F}^{-1}=\frac{1}{N_{c}}\left(\begin{array}{ccccc}
1 & 1 & 1 & 1 & 1 \\
1 & w^{-1} & w^{-1} & \ldots & w^{-\left(N_{c}-1\right)} \\
1 & w^{-2} & w^{-4} & \ldots & w^{-2\left(N_{c}-1\right)} \\
1 & \vdots & \vdots & & \vdots \\
1 & w^{-\left(N_{c}-1\right)} & w^{-2\left(N_{c}-1\right)} & \ldots & w^{-\left(N_{c}-1\right)^{2}}
\end{array}\right)_{(11)}
$$


Where,

$w=e^{\frac{-j 2 \pi}{N_{c}}}$

Second, to shelter the signal from the ISI (Inter-Symbol Interference) we add the cyclic prefix (CP) or what are commonly called the guard interval, we can express this step mathematically by multiplying the signal $\mathrm{z}$

$$
\xi=\left(\left(\begin{array}{cc}
0 & I_{N g} \\
& I_{N C}
\end{array}\right) \otimes I_{N t}\right)
$$

Where I is i.i.d matrix.

Eventually we transmit a OFDM symbol xp NcNt, 1 over a selective correlated Rayleigh fading channel $\mathrm{H}$, thus:

$$
y=H \cdot \xi_{1} \cdot \sqrt{N_{c}} \cdot\left(\mathrm{F}^{-1} \otimes I_{N t}\right) \cdot x_{N_{c} N_{t}, 1}^{p}+\omega
$$

Where $\mathrm{w}$ is an $\mathrm{Nt}(\mathrm{Nc}+\mathrm{Ng})$ white Gaussian noise vector. This calculation fits either with Joint Coding (JC) or Per Antenna Coding (PAC) technique. In fact, in the (JC) method, the information bit stream is first encoded and then converted into Nt parallel sub-streams of which each is modulated and mapped onto corresponding antenna. Fig 1 illustrates the (JC) scheme. However in (PAC) scheme, the incoming bit stream is first transformed to Nt parallel sub-streams and then encoding is performed per sub-stream. So, basically, the transmitter consists of Nt OFDM transmitters among which the information bits are multiplexed. At the receiver we consider the system is coherent over a selective correlated Rayleigh fading MIMO channel. First, the cyclic prefix is removed. This is done by discarding the first $\mathrm{NgNr}$ samples of $\mathrm{y}$,

$$
\hat{y}=\frac{1}{N_{c}}\left(\mathrm{~F} \otimes \mathrm{I}_{N_{r}}\right) \xi_{2} y
$$

Where $£ 2$ is defined as $\left[0 \mathrm{NcNgI}_{\mathrm{Ng}}\right]$ matrix. Second, the FFT is performed. Together, give results as

$$
x_{N_{c} N_{t}, 1}^{p}=\left\{\left(\mathrm{F} \otimes \mathrm{I}_{N_{t} N_{r}}\right) \xi_{3}\left(\mathrm{~F}^{-1} \otimes \mathrm{I}_{N_{t} N_{r}}\right)\right\}^{-1} \hat{y}
$$

Where $£ 3$ is commonly called the circulant matrix defined as:

$$
\xi_{3}=\xi_{2} H \xi_{1}
$$

The decision vector for each four symbols is then decoded at time $\left(\mathrm{n}_{\mathrm{i}}, \mathrm{n}_{\mathrm{i}+1}\right)$ using a sub-optimum decoder like a Zero Forcing or MMSE decoder. In the optimum decoder for the algebraic space time code was the Shnorr-Echnerr or Sphere-Decoder, but the the ZF or the MMSE still a good candidate for such codes, because they reduce the computational load regarding the Shnorr-Echnerr or the Sphere-Decoder without significant performance loss:

$$
\hat{v}=\phi_{p}^{-1} \times \hat{x}_{4,1}^{p}
$$

Where,

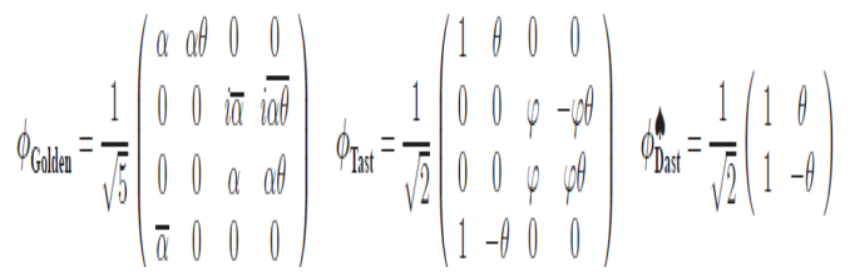

In this case we decode each 2 symbols together, thus we slice the received $\mathrm{x}_{4,1}$ Dast into $\mathrm{x}_{2,1}{ }^{\text {Dast }}[7,9,10]$.

\section{A. Continuous Policy}

\section{Adaptive Modulation}

To obtain the optimum CC-A adaptation policy for MIMO multiplexing we have to tackle a calculus of variations problem with two isoperimetric constraints. We denote by $f_{R i}\left({ }^{\lambda} \lambda\right), f_{S i}(\lambda): R^{m} \rightarrow R$ any nonnegative rate and power candidate adaptation laws for the ith eigen channel and by $\mathrm{R}_{\mathrm{i}}\left({ }^{\wedge} \lambda\right)$ and $\mathrm{S}_{\mathrm{i}}\left({ }^{\wedge} \lambda\right)$ the optimum laws i.e. 
those that maximize the ASE. The power laws are normalized to the target average power. ST. Mathematically, the MIMO multiplexing design problem is expressed as follows.

$$
\max _{\left\{\mathrm{f}_{R i}\right\},\left\{\mathrm{f}_{S i}\right\}} E_{\lambda}\left[\sum_{\lambda}^{m} f_{R i}(\hat{\lambda})\right](\mathrm{ASE})
$$

Subject to

$$
\begin{aligned}
& -1+E_{\hat{\lambda}}\left[\sum_{i=1}^{m} f_{S i}(\hat{\lambda})\right]=0 \\
& E_{\hat{\lambda}}\left[\sum_{i=1}^{m} f_{R i}(\hat{\lambda})\left(1-\beta_{i}\left(\hat{\lambda}, f_{R 1}, \ldots \ldots ., f_{R m}, f_{S 1}, \ldots \ldots ., f_{S m}\right)\right)\right]=0
\end{aligned}
$$

Where the conditional BER (normalized to the target BER, BERT) for the ith Eigen channel is defined as

$$
\beta i=\frac{1}{\overline{B E R_{T}}} E_{H}\left[\mathrm{BER}_{i}(\hat{\lambda}, \mathrm{H}) \mid \hat{\lambda}\right]
$$

With BERi $\left({ }^{\wedge} \lambda, \mathrm{H}\right)$ the instantaneous BER given the predicted and the true CSI. Under the Gaussian approximation the conditional BER can be computed from the signal-to-noise plus interference ratio (SINR), thus, using the usual exponential expression for MQAM,

$$
\beta_{i} \approx \frac{1}{5 \overline{B E R_{T}}} E_{H}\left[\exp \left(-\frac{8}{5} \frac{\operatorname{SINR}_{i}(\hat{\lambda}, \mathrm{H})}{2^{f_{R i}(\hat{\lambda})}-1}\right)\right]
$$

It will be shown at the end of this section that this Gaussian approximation is quite accurate due to the particular form of the optimum adaptive policy. Introduction and after some algebra it is straightforward to obtain

$$
\operatorname{SINR}_{i}(\hat{\lambda}, \mathrm{H})=\frac{\left|\sqrt{\hat{\lambda}_{i}}+\hat{\Upsilon}_{i i}\right|^{2} f_{S i}(\hat{\lambda})}{\sum_{j \neq i}\left|\hat{\Upsilon}_{i j}\right|^{2} f_{S j}(\hat{\lambda})+1 / \hat{\Upsilon}}
$$

With ${ }^{\wedge} \mathbf{Y} \sim{ }^{\wedge} \boldsymbol{\Xi}$ and the average SNR defined as $\mathrm{y}=\mathrm{ST} / \sigma 2 \mathrm{n}$.According to Appendix A, the conditional BER expression in (6) can be accurately computed by (8) at the top of the page, where xk are the zeros of the NP theorder Laguerre polynomial and Lxk the associated weight factors used for Gauss-Laguerre quadrature integration. Specifically, in expression $\Lambda \mathrm{j}=1$ for $\mathrm{m}=2$ and for $\mathrm{m}>2$

$$
\Lambda_{j}=\prod_{l=1, l \neq i, j}^{m} \frac{f_{s j}(\hat{\lambda})}{f_{s j}(\hat{\lambda})-f_{s l}(\hat{\lambda})}
$$

Which must be interpreted as a limit1 when $f S l=f S j$. To perform expectation over the predicted channel gain ${ }^{\wedge} \lambda$, note that ${ }^{\wedge} \lambda=(1-\chi) \xi$ with $\boldsymbol{\xi}$ the $m$-dimensional vector $\boldsymbol{\xi}=(\xi i)$ of unordered eigen values of $1 /(1-\chi)^{\wedge} \mathbf{H}{ }^{\wedge} \mathbf{H}$ $H \sim \mathbf{H H} H$. Consequently, the joint probability density function (pdf) $p^{\wedge} \lambda\left({ }^{\wedge} \lambda\right)$ is easily obtained from the Wishart pdf $p \xi(\xi)$ given in which can be expressed as:

$$
p_{\xi}(\xi)=\frac{1}{m !} \sum_{a, b}(-1)^{\operatorname{per}(\mathrm{a})+\operatorname{per}(\mathrm{b})} \prod_{i} \sum_{j=d}^{a_{i}+b_{i}-2+d} A_{j}\left(\mathrm{a}_{i}, \mathrm{~b}_{i}\right) \xi_{i}^{j} e^{-\xi i}
$$

Where $d .=|N T-N R|, \mathbf{a}=(\mathrm{a} i)$ and $\mathbf{b}=(\mathrm{b} i)$ represent permutation vectors of $\{1, \ldots, m\}$, the function per $(\cdot)$ is 0 or 1 , respectively, depending on whether the permutation is even or odd, and $A j(\mathrm{a} i$, bi $)$ is defined as the $(j+1)$ th coefficient of the following polynomial:

$x^{d} \sqrt{\frac{\left(\mathrm{a}_{i}-1\right) !\left(\mathrm{b}_{i}-1\right) !}{\left(\mathrm{a}_{i}-1+\mathrm{d}\right) !\left(\mathrm{b}_{i}-1+\mathrm{d}\right) !}} L_{a_{i}-1}^{d}(\mathrm{x}) \mathrm{L}_{b_{i}-1}^{d}(\mathrm{x})$ 
Where $\operatorname{Ldn}(x)$ is the generalized Laguerre polynomial. It is shown in that the marginal pdf $p \xi(\xi)$ can be represented as follows:

$$
p_{\xi}(\xi)=\mathrm{e}^{-\xi} \sum_{j=d}^{2(\mathrm{~m}-1)+\mathrm{d}} B_{j} \xi^{j}
$$

With $B j$ defined as the $(j+1)$ Th coefficient of the following polynomial

$$
\frac{1}{m} \sum_{i=1}^{m} x^{d} \frac{(\mathrm{i}-1) !}{(\mathrm{i}-1+d) !}\left(\mathrm{L}_{i-1}^{d}(\mathrm{x})\right)^{2}
$$

In general, solving the problem stated is hard due to the coupling between Eigen channels introduced through both the conditional BER (imperfect CSI induced interference) and the statistical dependence between the components of $\lambda^{\lambda} \lambda$. However, under certain approximations it is possible to find an accurate closed-form adaptation policy for MIMO multiplexing with an average BER constraint and imperfect CSI. To analyze the behavior of optimum A-QAM MIMO multiplexing we distinguish two scenarios according to the quality of the available CSI: good quality ( $\chi$ relatively small) and bad quality ( $\chi$ relatively high). $[11,12]$

\section{Results And Disscussions}

As Fig 2 show how ergodic capacity change with respect to SNR value and number of transmitting antenna $\left(\mathrm{n}_{\mathrm{t}}\right)$. here we use MATLAB SIMULINK R2010 for calculating Ergodic capacity. If we compare our result with first reference paper result than there is good improvement in Ergodic capacity when using less number of antenna but when we using more number of transmit antenna then at very small value of SNR , ergodic capacity increase rapidly. Hence we are able to overcome the limitation of ergodic capacity with small number of antenna by using ASTC encoder. We see that at $n_{t}=1$ when we increase SNR the value of ergodic capacity also increase w.r.t. SNR. It does not come study state as in the result of first reference paper. Fig number 3 shows the individual variation of ergodic capacity with number of transmit antenna. When number of transmit antenna $n_{t}=30$ its value above the 120 which mean that we enhance the channel capacity by using ASTC encoder and adaptive QAM

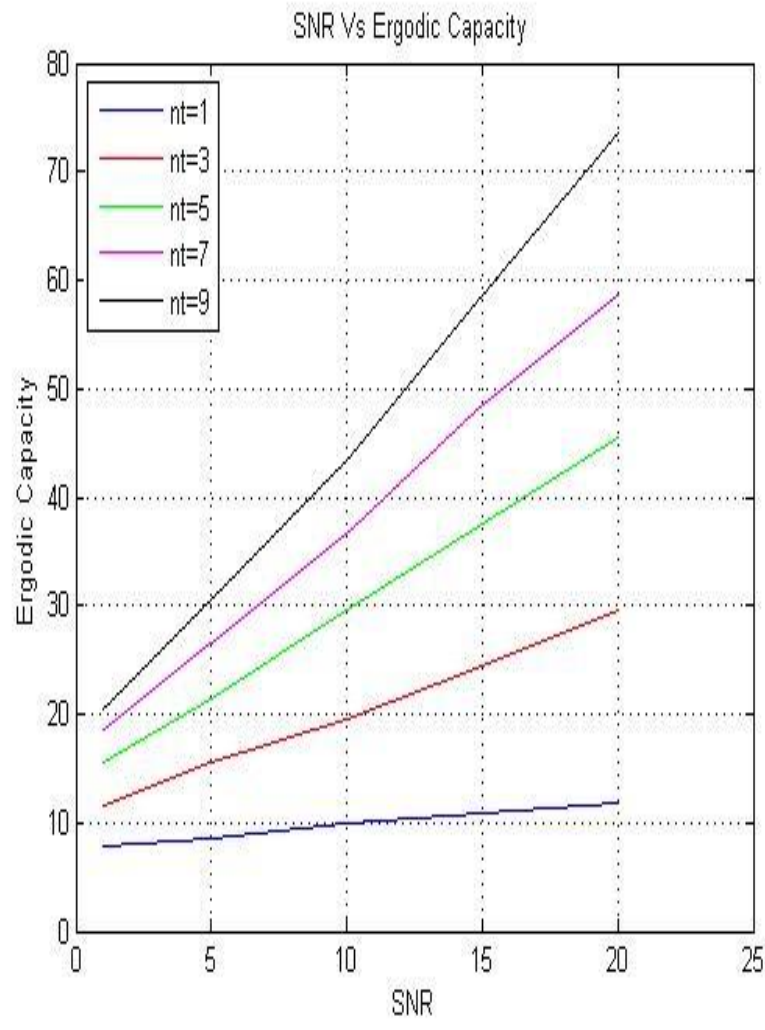

Fig. 2 SNR versus Ergodic Capacity 


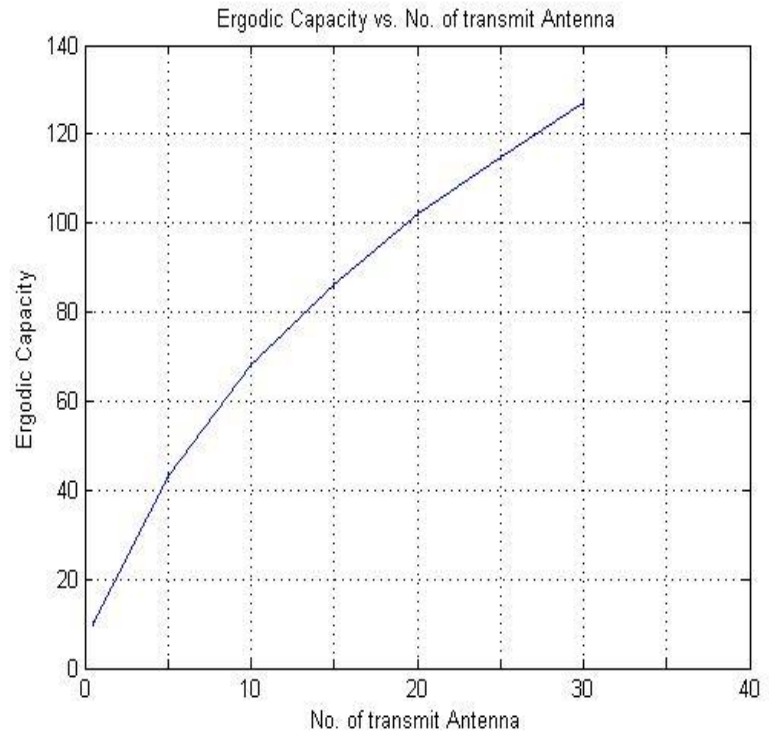

Fig. 3: Ergodic capacity versus no. of transmit antenna

In Fig 4. The y axis vertices variable 10-0 actually represent the $10^{-0} \mathrm{BER}$ and so on. If we increases the SNR then BER is reduce it also shows in figure when SNR increases the value of BER decrease. At the 20 SNR the value our BER is below the $10^{-30}$ which tell us that we improve the system performance. In Figure 5, we have a plot of the spectral efficiency of adaptive modulation versus average SNR in dB. We do not take into account whether or not the bits are the correct ones that were sent or not. Because we have set the target BER to a value that we believe the system must operate under, the adaptation system will try to achieve that level of performance Note that at low SNR value, the system achieves 2 bits per symbol, and QPSK is primarily used. However, when the SNR increases, the throughput also improve steadily, which indicates that we are beginning to use more spectrally efficient modulation schemes. The curve begins to level out at close to $30 \mathrm{~dB}$, as 64QAM becomes the modulation scheme used most often and QPSK is rarely used. when SNR improves, the system is more able to choose more efficient modulation schemes by using adaptive QAM. Fig. 6 shows the performance of system by using ASTC encoder and without also tell that by using ASTC encoder we increase our system capacity w.r.t. SNR vs BER graph analysis.

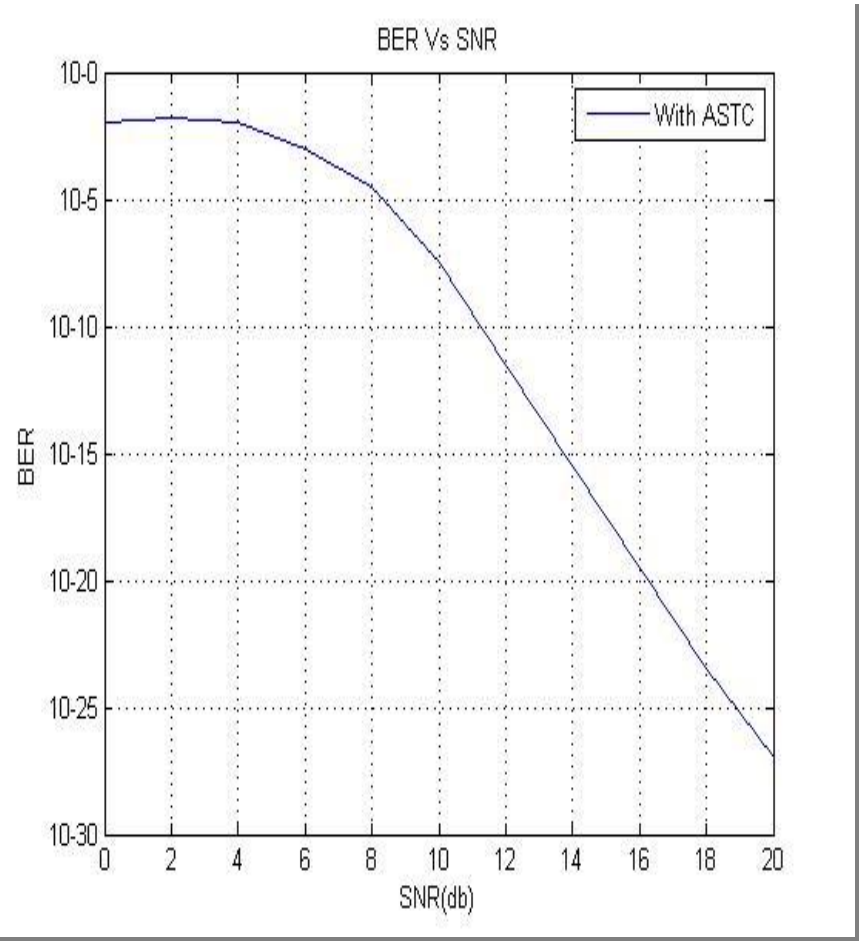

Fig. 4 System performance w.r.t. SNR vs. BER 


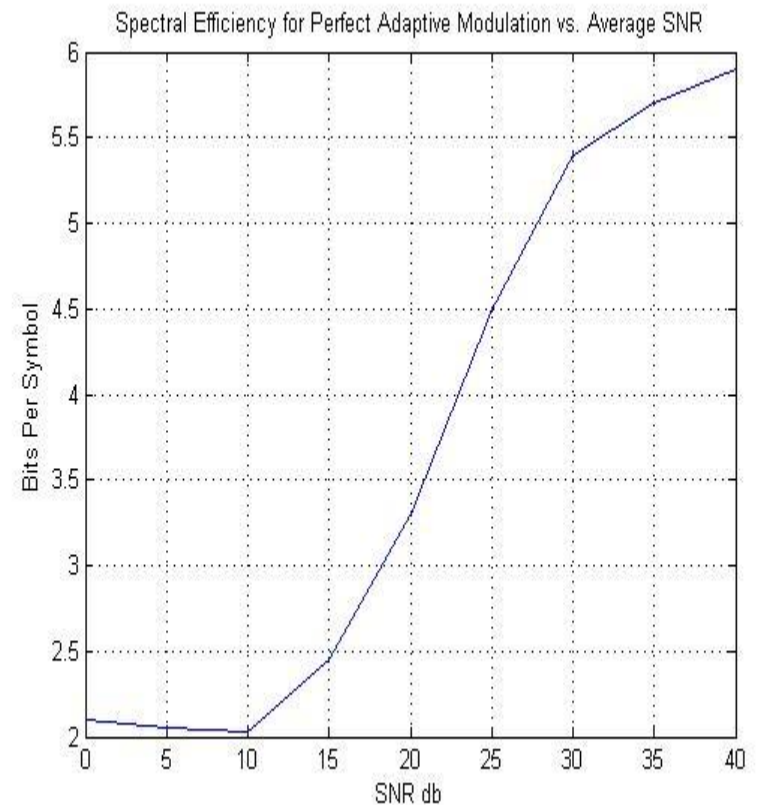

Fig. 5 Spectral Efficiency for Perfect Adaptive Modulation vs. Average SNR for a Rayleigh Channel

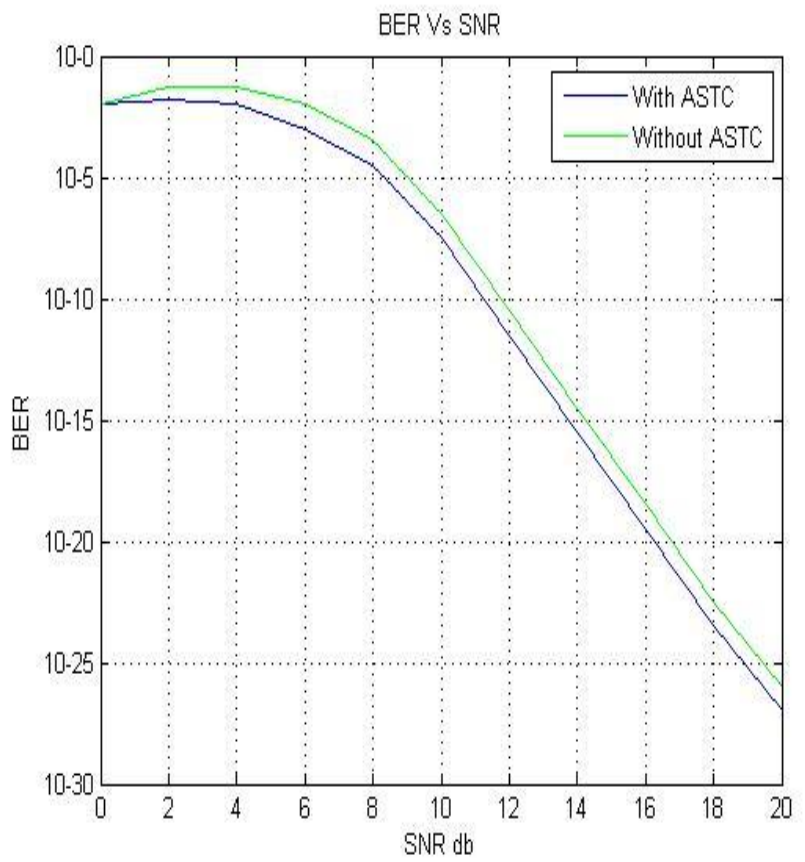

Fig. 6 System performance by using ASTC encoder w.r.t. SNR vs BER

\section{Conclusion}

Ergodic channel capacity has some limitation in MIMO OFDM system therefore it is necessary to improve this because it affects the system performance. To improve this we use ASTC encoder because it has properties full rank full, full rate, and non vanishing determinant for increasing rate. ASTC is also able to reduce the major difficulty of OFDM's Large Peak to Average Power Ratio (PAPR).As a result we find that the ASTC codes like a good compromise between a PAPR reduction scheme and BER performance. Our results also show that adaptive modulation for MIMO OFDM system is much more sensitive to imperfect CSI that MIMO beam forming. We can analyze MIMO-OFDM system and use various algorithms to optimize channel capacity.

\section{Acknowldgement}

I would like to thank my friends Anupam Kumar ,Amandeep, Ajay Sharma, Manoj Kumar, Kapil Sharma, Pankaj Sharma, Abhradip Paul and all my family member and my teachers who help me. 


\section{References}

[1] Prof. Jaiswal and Er. Kumar, Anil. And Singh, Anand Prakash. (2012) "performance analysis of mimo-ofdm in Rayleigh fading channel" international journal of scientific and research publication vol.2 issue 5, May 2012

[2] Salim, Alouini Mohamed. And J Goldsmith, Andrea. Member IEEE (1999),"Capacity of Rayleigh Fading Channel under Different Adaptive Transmission and Diversity-Combining Techniques".

[3] Xiao, chengshan. and Zheng, Yahang R." Ergodic Capacity, Capacity Distribution and Outage Capacity of MIMO Time-Varying and Frequency-Selective Rayleigh Fading Channels" Department of Electrical \& Computer Engineering University of Missouri, Columbia, MO 65211, USA.

[4] Gupta, Jishu Das. And Ziri-castro, Karla. and Suzuki, Hajime.( 2007). "Capacity Analysis of MIMO OFDM Broadband Channels In Populated Indoor Environments," in proceedings of IEEE International Symposium on Communications and Information Technologies, Oct. 17-19,Sydney, pp. 273-278.

[5] Bolcskei, Helmut. And Gesbert, David. and Paulraj, Arogyaswami J. Member, IEEE. (2002)" On the Capacity of OFDM-Based Spatial Multiplexing Systems" IEEE TRANSACTIONS ON COMMUNICATIONS, VOL. 50, NO. 2 , FEBRUARY 2002.

[6] Ahmed Bannour, Mohamed Lassaad Ammari, Yichuang Sun, Ridha Bouallegue,2011 “ On the Capacity of ASTC-MIMO-OFDM System in a Correlated Rayleigh Frequency-Selective Channel” 978-1-4244-8331-0/11,2011 IEEE.

[7] Ahmad Bannour, Mohamed Lassaad Anmmari and Ridha Bouallegue,2010 "Adaptation of ASTC in a Correlated Rayleigh Frequency-Selective Fading Channels in OFDM systems with PAPR Reduction" International Journal of Wireless \& Mobile Networks (IJWMN), Vol.2, No.2, May 2007.

[8] J. C. Belfiore, G. Rekaya, and E. Viterbo, "The golden code: a 2 x 2 full-rate space-time code with non-vanishing determinants," IEEE Trans. Inform. Theory, vol. 13, pp. 67-75, 2004.

[9] A. Bannour, M. L. Ammari, and R. Bouallegue, "Analysis of ASTC in a correlated rayleigh fading channel with imperfect channel estimation," International Conference on Advanced Communication Technology, 2010[10]

[10] M. L. Ammari and F. Gagnon, "Iterative channel estimation and decoding of turbo-coded OFDM symbols in selective Rayleigh channel," Canadian J. Elect. Comput. Eng., vol. 32, no. 1, pp. 9-18, 2007.

[11] Jos' e F. Paris and Andr [10] M. L. Ammari and F. Gagnon, "Iterative channel estimation and decoding of turbo-coded OFDM symbols in selective Rayleigh channel,"

[12] R. Weinstock, Calculus of Variations, 1st ed., McGraw-Hill, New York, 1952 